\title{
Correction to: The accuracy and reliability of digital measurements of gingival recession versus conventional methods
}

Hytham N. Fageeh*, Abdullah A. Meshni, Hassan A. Jamal, Reghunathan S. Preethanath and Esam Halboub

\section{Correction to: BMC Oral Health (2019) 19: 154 \\ https://doi.org/10.1186/s12903-019-0851-0}

After publication of the original article [1], the authors reported that an author's name has been mispelled. "Helboub" should be replaced with "Halboub".

The original article has been updated as well.

Published online: 30 August 2019

\section{Reference}

1. Fageeh, et al. BMC Oral Health. 2019;19:154. https://doi.org/10.1186/s12903019-0851-0.

* Correspondence: hfageeh@jazanu.edu.sa

Division of Periodontics, College of Dentistry, Jazan University, P.O.Box 114, Jazan 45142, Saudi Arabia 